\title{
Non-invasive assessment of tumor PD-L1 status with circulating tumor cells
}

\author{
Bryan C. Ulrich ${ }^{1}$, Nicolas Guibert ${ }^{2}$ \\ ${ }^{1}$ Emory School of Medicine, Atlanta, GA, USA; ${ }^{2}$ Thoracic Oncology Department, Larrey Hospital, University Hospital of Toulouse, Toulouse, \\ France \\ Correspondence to: Nicolas Guibert. Thoracic Oncology Department, Larrey Hospital, University Hospital of Toulouse, Toulouse, France. \\ Email: guibert.n@chu-toulouse.fr. \\ Comment on: Dhar M, Wong J, Che J, et al. Evaluation of PD-L1 expression on vortex-isolated circulating tumor cells in metastatic lung cancer. Sci \\ Rep 2018;8:2592.
}

Submitted Sep 20, 2018. Accepted for publication Sep 30, 2018.

doi: 10.21037/atm.2018.10.09

View this article at: http://dx.doi.org/10.21037/atm.2018.10.09

\section{Introduction}

Now FDA-approved in the treatment of over ten malignancies, checkpoint blockade agents have transformed the treatment of cancer. These agents work by inhibiting the PD-1/PD-L1 interaction between cytotoxic T cells and tumor cells, an interaction which promotes immune evasion. PD-L1 expression levels by tumor cells, as assessed by tissue biopsy, is currently the most well-validated and clinically used predictive biomarker of response to checkpoint blockade agents $(1,2)$. However, PD-L1 level is known to be an imperfect biomarker, and some FDA approvals do not set a PD-L1 expression level threshold to determine patient candidacy for checkpoint blockade.

Challenges regarding PD-L1 expression as a biomarker fall into two categories. First, PD-L1 expression is an inexact determinant of response to checkpoint blockade agents. Although levels broadly correlate to response rates, some patients with absent tumor PD-L1 expression on tissue histology do respond to these agents, while some patients with high PD-L1 levels do not respond. Several new biomarkers of response have arisen in an attempt to better predict response, including tumor mutational burden, tumor immune-profile, and gender (3-7). However, further validation of these is required before widespread clinical adoption. Second, PD-L1 levels must be assessed by immunohistochemistry (IHC) of tumor tissue, thus requiring tissue biopsy. With an ever-increasing armamentarium of targeted agents, in addition to dynamic therapeutic strategies which may indicate multiple tissue biopsies over the course of a patient's treatment history, the need for non-invasive biomarkers is clear and growing. Cell-free DNA (cfDNA) has found clinical success as a biomarker of response and resistance in various clinical situations $(8,9)$. However, a non-invasive biomarker to predict response to checkpoint blockade agents has been elusive.

Dhar et al. sought to address both categories of the challenges posed by PD-L1 as a predictive biomarker of response with their investigation into $\mathrm{PD}-\mathrm{L} 1$ expression on circulating tumor cells (CTCs) in metastatic nonsmall cell lung cancer (NSCLC) patients (10). First, as circulating biomarkers (CTCs and cfDNA alike) are thought to represent the entirety of a tumor, including its metastatic lesions, PD-L1 expression on CTCs could potentially shed light on the imperfections of PD-L1 as a predictive biomarker. Indeed, cfDNA has been shown, in some instances, to better capture the genetic heterogeneity of tumors than a tissue biopsy $(11,12)$. It is possible that PD-L1 expression heterogeneity is not fully captured by the small piece of tissue that is analyzed via IHC, and that CTCs more accurately represent tumor PD-L1 expression on the whole. Second, as CTCs can be collected noninvasively, using them to assess PD-L1 expression level overcomes limitations to the use of tissue PD-L1 level; it spares patients another biopsy. Thus, Dhar and colleagues attempt not just to extend the advantages of all non-invasive biomarkers into the territory of predictive biomarkers to checkpoint blockade, but also to provide a tool that 
potentially aids in understanding PD-L1 levels on tumor cells throughout the body, thereby improving predictions of therapeutic response to checkpoint blockade.

While this effort deserves praise for its scope, assay performance concerns and, more importantly, questions surrounding PD-L1 heterogeneity and what it means in the context of predicting response to checkpoint blockade hinder the formation of strong conclusions until further research and development into this technology.

\section{PD-L1 expression on "CTCs"}

While a large part of the work by Dhar and colleagues is dedicated to developing protocols to assess PD-L1 expression on CTCs, questions remain. To isolate and assess CTCs, they use a size-based CTC capture and an immunofluorescence (IF) stain to quantify PD-L1 expression levels. Of note, their assay only detects CTCs in $45 \%$ of patients with metastatic NSCLC. This is a major issue, as it would significantly limit the ability of this technology to provide any information for more than half of patients with metastatic NSCLC. Furthermore, the observation of CD45-/DAPI+ cells with "cytomorphological patterns of malignancy" in all healthy donors' blood, called "CTCs" by the authors is really surprising, and obviously raises question about the nature of "CTCs" detected in cancer patients. Of the 14 samples that were positive for CTCs, 12 had any PD-L1 positive CTCs. Certain data has shown discordance between PD-L1 expression level on biopsies and matched resected tumor, potentially suggesting that these latter small samples underestimate PD-L1 status due to tumor heterogeneity (13). In agreement with this observation, almost all patients have $\mathrm{PD}-\mathrm{L} 1$ positive CTCs $(14,15)$, which further complicates the utility of this assay. The threshold used by Dhar and colleagues is misleading, because the PD-L1 expression assay was not run on "CTCs" collected from healthy individuals.

Although we may speculate from this data that PDL1 positive CTCs will be poorly sensitive, their attempts to correlate it with tumor PD-L1 status consists of only 4 comparisons, and thus is underpowered. Unfortunately, no conclusion can be made from this comparison, and further correlative studies will be needed. Until these studies are completed, any statement of potential clinical utility would be unjustified. In other studies, except for one team (16), none of the research investigating concordance between tissue and CTCs PD-L1 expression demonstrated a strong quantitative correlation $(14,15,17)$. Spatial (13) and temporal (18-20) tumor heterogeneity has been proposed as a potential explanation for these discrepancies, however the very limited number of CTCs isolate probably also preclude a valuable comparison.

\section{Heterogeneity in PD-L1 expression}

As stated earlier, the authors claim that the pool of CTCs may better represent the PD-L1 status of lesions throughout the body than the spatially-limited tumor biopsy. Evidence that tumor heterogeneity is clinically meaningful is evident in genetic analyses of re-biopsy tissue in patients who are progressing to first-line EGFR tyrosine kinase inhibition in NSCLC (11). The false negative rate of tissue biopsy in identifying genomic causes of progression is well documented, and cfDNA is now used in this setting as it may identify when tissue biopsy does not capture the presence of a targetable mechanism of resistance (11). Thus, the idea that blood-based biomarkers can, in some situations, depict a more accurate representation of a given tumor characteristic throughout the body has merit.

A patient with a tumor biopsy that detects any amount of PD-L1 expression in a given tumor for which checkpoint blockade is approved, will be given checkpoint blockade, rendering a blood-based assay irrelevant. Considering now a situation in which a patient's tumor biopsy shows a negative PD-L1 result: It is in this situation that the authors believe a CTC assay may provide more accurate predictive capabilities than tissue, by assuming that tissue PD-L1 analysis underestimates true PD-L1 expression. This hypothesis is interesting, and answering it will entail comparing, through prospective studies, outcomes to checkpoint blockade in patients with PD-L1 positive tissue versus in those with PD-L1 negative tissue but PD-L1 positive CTCs. If these response rates are similar, then we know that tissue is indeed calling false negatives on some patients that would benefit. Overall, further investigation into PD-L1 expression on CTCs is required to understand its ability to inform clinical practice as a predictive biomarker of response in the case that a tissue biopsy is negative for PD-L1 expression. So far, none of the three studies that correlated PD-L1 expression on CTCs and outcomes under PD1 inhibitors could clearly establish this approach as a potential biomarker. In two of them, the rate of PD-L1 positive CTCs was very high and of bad prognosis but the absence of control arm of patients treated by chemotherapy precluded a predictive value analysis $(14,15)$, while in this work, the number of patients 
was too low to draw definitive conclusions. Another pitfall to this approach is the cost and turnaround time of this cumbersome technique.

\section{Conclusions}

Currently, patient candidacy to receive checkpoint blockade in some clinical situations is based on tissue IHC for PD-L1 expression, while other indications do not require a PD-L1 expression level. Although it is the most clinically well-validated predictive biomarker of response to checkpoint blockade, PD-L1 expression is imperfect and investigation into other biomarkers is ongoing. In their manuscript, Dhar and colleagues develop a method of capturing and assessing CTCs for PD-L1 expression, yet assay performance issues need to be addressed before continued investigation. Altogether, accumulating data now question the clinical relevance of CTCs in the immunotherapy space, as this cumbersome, costly and poorly sensitive approach has failed so far to predict outcomes under PD1 inhibitors $(14,15)$. The development of non-invasive biomarkers to predict response to checkpoint blockade should remain a top priority for researchers as it may lead to insights into tumor biology and immunology as well as provide tools to improve our ability to target therapies to patients most likely to benefit. Estimation of the tumor mutation burden using plasma whole exome sequencing, or targeted next generation sequencing looking for predefined genomic events known to be associated to outcomes under immune checkpoint inhibitors seem like more appealing approaches (21-24).

\section{Acknowledgements}

None.

\section{Footnote}

Conflicts of Interest: The authors have no conflicts of interest to declare.

\section{References}

1. Hirsch FR, McElhinny A, Stanforth D, et al. PDL1 Immunohistochemistry Assays for Lung Cancer: Results from Phase 1 of the Blueprint PD-L1 IHC Assay Comparison Project. J Thorac Oncol 2017;12:208-22.

2. Khunger M, Hernandez AV, Pasupuleti V, et al.
Programmed Cell Death 1 (PD-1) Ligand (PD-L1)

Expression in Solid Tumors As a Predictive Biomarker of Benefit From PD-1/PD-L1 Axis Inhibitors: A Systematic Review and Meta-Analysis. JCO Precis Oncol 2017. DOI: 10.1200/PO.16.00030

3. Rizvi NA, Hellmann MD, Snyder A, et al. Cancer immunology. Mutational landscape determines sensitivity to PD-1 blockade in non-small cell lung cancer. Science 2015;348:124-8.

4. Hellmann MD, Ciuleanu TE, Pluzanski A, et al. Nivolumab plus Ipilimumab in Lung Cancer with a High Tumor Mutational Burden. N Engl J Med 2018;378:2093-104.

5. Ulrich BC, Guibert N. Immunotherapy efficacy and gender: discovery in precision medicine. Transl Lung Cancer Res 2018;7:S211-3.

6. Conforti F, Pala L, Bagnardi V, et al. Cancer immunotherapy efficacy and patients' sex: a systematic review and meta-analysis. Lancet Oncol 2018;19:737-46.

7. Lizotte PH, Ivanova EV, Awad MM, Jones RE, Keogh L, Liu H, et al. Multiparametric profiling of non-smallcell lung cancers reveals distinct immunophenotypes. JCI Insight 2016;1:e89014.

8. Ulrich BC, Paweletz CP. Cell-Free DNA in Oncology: Gearing up for Clinic. Ann Lab Med 2018;38:1-8.

9. Alix-Panabières C, Pantel K. Clinical Applications of Circulating Tumor Cells and Circulating Tumor DNA as Liquid Biopsy. Cancer Discov 2016;6:479-91.

10. Dhar M, Wong J, Che J, et al. Evaluation of PD-L1 expression on vortex-isolated circulating tumor cells in metastatic lung cancer. Sci Rep 2018;8:2592.

11. Oxnard GR, Thress KS, Alden RS, et al. Association Between Plasma Genotyping and Outcomes of Treatment With Osimertinib (AZD9291) in Advanced Non-SmallCell Lung Cancer. J Clin Oncol 2016;34:3375-82.

12. Murtaza M, Dawson SJ, Tsui DW, et al. Non-invasive analysis of acquired resistance to cancer therapy by sequencing of plasma DNA. Nature 2013;497:108-12.

13. Ilie M, Long-Mira E, Bence C, et al. Comparative study of the PD-L1 status between surgically resected specimens and matched biopsies of NSCLC patients reveal major discordances: a potential issue for anti-PD-L1 therapeutic strategies. Ann Oncol 2016;27:147-53.

14. Guibert N, Delaunay M, Lusque A, et al. PD-L1 expression in circulating tumor cells of advanced nonsmall cell lung cancer patients treated with nivolumab. Lung Cancer 2018;120:108-12.

15. Nicolazzo C, Raimondi C, Mancini M, et al. Monitoring 
PD-L1 positive circulating tumor cells in non-small cell lung cancer patients treated with the PD-1 inhibitor Nivolumab. Sci Rep 2016 24;6:31726.

16. Ilié $M$, Szafer-Glusman E, Hofman V, et al. Detection of PD-L1 in circulating tumor cells and white blood cells from patients with advanced non-small-cell lung cancer. Ann Oncol 2018;29:193-9.

17. Kallergi G, Vetsika EK, Aggouraki D, et al. Evaluation of PD-L1/PD-1 on circulating tumor cells in patients with advanced non-small cell lung cancer. Ther Adv Med Oncol 2018;10:1758834017750121.

18. Han JJ, Kim DW, Koh J, et al. Change in PD-L1 Expression After Acquiring Resistance to Gefitinib in EGFR-Mutant Non-Small-Cell Lung Cancer. Clin Lung Cancer 2016;17:263-70.e2.

19. Gong X, Li X, Zhou C. 81P Radiation resistance induced immunity evasion by evoking PD-L1 expression. J Thorac Oncol 2016;11:S89-90.

20. Lim SH, Hong M, Ahn S, et al. Changes in tumour expression of programmed death-ligand 1 after neoadjuvant concurrent chemoradiotherapy in patients

Cite this article as: Ulrich BC, Guibert N. Non-invasive assessment of tumor PD-L1 status with circulating tumor cells. Ann Transl Med 2018;6(Suppl 1):S48. doi: 10.21037/ atm.2018.10.09 with squamous oesophageal cancer. Eur J Cancer 2016;52:1-9.

21. Guibert N, Jones G, Beeler JF, et al. Early prediction of outcomes to PD1 inhibitors in non-small cell lung cancer (NSCLC) using next generation sequencing (NGS) of plasma circulating tumor DNA (ctDNA). J Clin Oncol 2018;36:abstr 9078.

22. Davis AA, Chae YK, Agte S, et al. Comparison of tumor mutational burden (TMB) across tumor tissue and circulating tumor DNA (ctDNA). J Clin Oncol 2017;35:abstr e23028.

23. Fabrizio D, Lieber D, Malboeuf C, et al. A blood-based next-generation sequencing assay to determine tumor mutational burden (bTMB) is associated with benefit to an anti-PD-L1 inhibitor, atezolizumab. Cancer Res 2018;78:abstr 5706.

24. Gandara DR, Kowanetz M, Mok TS, et al. Blood-based biomarkers for cancer immunotherapy: Tumor mutational burden in blood (bTMB) is associated with improved atezolizumab (atezo) efficacy in 2L+ NSCLC (POPLAR and OAK). Ann Oncol 2017;28:abstr 1295O. 